\title{
Radiative transfer modeling of the dust disk of the Herbig Be star R Monocerotis
}

\author{
K. Murakawa \\ Max-Planck-Institut für Radioastronomie, Auf dem Hügel 69, 53121 Bonn, Germany \\ e-mail: murakawa@mpifr-bonn.mpg.de
}

Received 16 December 2008 / Accepted 8 July 2010

\begin{abstract}
Our previous near-infrared (NIR), high-resolution polarimetric images of the Herbig Be star R Mon show that the polarization disk has a large, nearly constant angular size of $\sim 4^{\prime \prime}$ in the $J H K$ bands, low polarizations of $1.1-1.5 \%$ in the $H$ and $K$ bands, and somewhat centro-symmetrical vector alignment. To derive the disk geometry parameters and grain sizes that explain this result, we modeled $\mathrm{R}$ Mon's dust disk by combining it with previously obtained spectral energy distribution and gas phase observations. Our model assumes an inner disk and outer envelope structure, dust of different populations for the disk and envelope, and a power-law size distribution of $n(a) \propto a^{-3.5}$ with $0.005 \mu \mathrm{m} \leq a \leq a_{\max } \mu \mathrm{m}$. We obtained an outer disk radius of 3000 AU, a ratio of the height to the radius of the disk of $H_{0}=0.4$, a $V$-band optical depth of 4.2 in the disk midplane, and an $a_{\max }$ of $1000.0 \mu \mathrm{m}$ for the disk. Such a large extension of the disk is similar to the ones of the central feature detected in previous observations in the CO emission lines (1500 AU) and the $2.7 \mathrm{~mm}$ continuum (3000 AU). The shape and angular size of the polarization disk in our model fit the observations and approximately trace the projected appearance of the real disk. Since the estimated dust disk height has an intermediate value and is lower than the gas phase (CO) disk height $\left(H_{0}=1.0\right)$, it is likely that the dust settling is ongoing. Our selected model with a large dust size with an $a_{\max }$ of $1000.0 \mu \mathrm{m}$ in the inner part of the disk and a disk mass of $0.02 M_{\odot}$ fit the near-infrared polarization towards the disk and the mid-infrared to millimeter fluxes well. Our estimated disk mass roughly agrees with a previous gas phase modeling of $\sim 0.01 M_{\odot}$. Such a large grain model reproduces low NIR polarizations (1.6\%), which is consistent with our observation. Not detecting the vector alignment is explained with this large grain model and an intermediate optical depth of the disk. We expect that R Mon's disk contrasts to the typical T Tauri and Herbig Ae disks in terms of an expected large outer disk radius and an intermediate optical depth. Evidence of the dust settling and large grains suggests that the dust in R Mon's disk grows faster than the disk accretion advances, which is theoretically predicted in typical T Tauri disks.
\end{abstract}

Key words. circumstellar matter - reflection nebulae - infrared: ISM

\section{Introduction}

It is widely accepted that the disk is an important component of young stellar objects (YSOs) because its structure and dust properties reflect the physics of star formation, and it is a field for planet formation. In the past 20-30 years, T Tauri disks have been intensively studied (e.g., Adams \& Shu 1986; Kenyon \& Hartmann 1987; Bertout et al. 1988; Calvet et al. 1994; Chiang \& Goldreich 1997). Their radiative transfer analyses and hydrodynamic treatments (see also Shakura \& Sunyaev 1973; Pringle 1981) predict flared disk structures and can explain spectral energy distributions (SEDs) in the mid- (MIR) and far-infrared (FIR) in many objects. Beckwith \& Sargent (1991) detected low dust opacity spectral indices $\beta$ in several T Tauri stars from their submillimeter observations. This is the first discovery of evidence for large grains in disks of these objects.

These analytical methods have also been applied to the intermediate-mass counterparts. In terms of the infrared SED, the Herbig $\mathrm{Ae} / \mathrm{Be}$ stars are classified into two groups (e.g. Meeus et al. 2001; Dullemond et al. 2001; Natta et al. 2001; Dullemond \& Dominik 2004a; Isella \& Natta 2005). Group I objects have a flat mid-infrared flux. The disks probably have a flared structure, similar to T Tauri stars. On the other hand, a significantly lower far-infrared flux is seen in Group II objects. In this object class, which is thought to be more evolved than the
Group I objects, the outer part of the disk is deposited into the midplane and is shaded from the radiation of the central star by the puffed-up inner rim. In addition, the dust is thought to grow in size in this advanced phase. In fact, in some objects, such as CQ Tau (Testi et al. 2003) and TW Hya (Wilner et al. 2005), evidence of large grains has been obtained from millimeter flux excess and $10 \mu \mathrm{m}$ spectra (e.g. van Boekel et al. 2003, 2005).

As these works have shown, investigations of disk properties benefit mainly from SED analyses in the thermal emission regime. On the other hand, other attempts by optical- to near-infrared (NIR) imaging polarimetry have been carried out (Bastien \& Ménard 1988, 1990; Kenyon et al. 1993; Whitney \& Hartman 1993; Fischer et al. 1994, 1996; Whitney et al. 1997; Lucas \& Roche 1997, 1998; Wood et al. 1998). In polarization images of YSOs seen nearly edge-on, what is called a polarization disk, where the polarization vectors are aligned in the equatorial direction, is often detected at the dark lane. This feature is reproduced with models where an optically thick dust disk or torus structure exists around the central star (Bastien \& Ménard 1988, hereafter BM).

Although a polarization disk does not directly indicate the real (dust) disk, i.e. a region with a Keplerian rotating matter within the centrifugal radius, detection of a polarization disk is, in fact, regarded as evidence of a disk according to the aforementioned robust works. However, the polarization analyses have 
been limited until recently, because the angular resolutions of the images were not high enough to resolve the disk, which causes pseudo polarization patterns. In addition, the BM scattering model is originally intended to explain the vector alignment in typical T Tauri disks. Disk properties for other object classes such as high-mass counterparts are more poorly understood. Recent high-resolution imaging techniques relieve the problem of the pseudo polarization pattern and allow us to improve our interpretations of YSO disks. In our recent work, we generalized the BM model and developed an analytical method for deriving information on the grain sizes and the disk geometry based on high-resolution imaging (Murakawa 2010, hereafter M10).

In our previous work, we obtained $J H K$-band polarimetric images of the Herbig $\mathrm{Be}$ star $\mathrm{R}$ Mon using the adaptive optics-equipped instruments VLT/NACO and Subaru/CIAO (Murakawa et al. 2008a, hereafter M08a). The important results are as follows. (1) The polarization disk has a butterfly shape with an $\sim 4$ " extension. This size does not change much in the $J H K$ bands. (2) The polarization in the polarization disk is as low as $1.1-1.5 \%$ in the $H K$ bands. The polarization vectors towards the polarization disk are not clearly aligned along the equatorial plane, but are somewhat centro-symmetric. A comparison with submillimeter/millimeter observations by Fuente et al. (2003, 2006, hereafter F06) is also interesting. The extension of the polarization disk is closer to the $\mathrm{CO}$ gas phase disk (1500 AU 4") and the central feature in the $2.7 \mathrm{~mm}$ continuum ( $3000 \mathrm{AU} \sim 8^{\prime \prime}$ ) than is detected both in the $1.3 \mathrm{~mm}$ continuum and an estimated disk radius of $150 \mathrm{AU}\left(\sim 0.0^{\prime} 4\right)$ by a previous modeling (Alonso-Albi et al. 2009). The disk height of the gas phase disk was estimated to be $1500 \mathrm{AU}$ at the outer disk radius of $1500 \mathrm{AU}$ (F06). In contrast, the appearance of the polarization disk looks thinner.

From the above results, some questions may rise: (1) what are the physical reasons for producing the low NIR polarizations and for not detecting the vector alignment; (2) what kind of disk properties are expected in R Mon from our analysis; and (3) is the dust distribution in the disk significantly different from the gas phase, i.e. do these results prove that the dust layer concentrates more towards the midplane than the gas phase molecules? To provide quantitative interpretations of R Mon's disk, we performed radiative transfer calculations. In our modeling, we take not only our previous NIR polarimetric images into account but also the previously observed SED and CO observations (F06) to better constrain the model parameters. We also apply our generalized scattering model for the R Mon case and study the physical conditions for producing the polarization disk, as questioned above. Our approach demonstrates the power of a complementary combination with an SED and polarization analyses and a gas phase observation. In Sect. 2, we describe the details of our modeling and present the results. In Sect. 3, we show the effects of the grain size in the disk and the disk geometry to justify our model results and discuss the properties of the R Mon disk.

\section{Radiative transfer modeling}

The stellar system of R Mon has been extensively studied. We adopted a distance of 800 pc (Jones \& Herbig 1982), a stellar mass of $10.4 M_{\odot}$, and an effective temperature of $30000 \mathrm{~K}$ (Hillenbrand et al. 1992). Cohen et al. (1984) estimated a luminosity of $1400 L_{\odot}$. Close et al. (1997) discovered a companion with a luminosity of $\sim 50 L_{\odot}$. Since this is much fainter than the primary, we assume that the spectrum of the radiation source has a single blackbody function with the aforementioned effective temperature and the luminosity of only the primary.
R Mon exhibits a complex monoconical lobe towards the north (Walsh \& Malin 1985; Lightfoot 1989; Close et al. 1997; Murakawa et al. 2008a). However, since our purpose is to study the dust disk and not the detailed appearance of the outer envelope, we apply an axi-symmetric geometry with an inner disk and infalling envelope structure.

For the disk, we examine two different forms of the mass density distribution (Shakura \& Sunyaev 1973; Lazareff et al. 1990; Fischer et al. 1996), which are given with the twodimensional cylindrical coordinate $(r, z)$ by

$$
\begin{aligned}
\rho_{\text {disk }}^{\mathrm{F} 1}(r, z) & =\rho_{\mathrm{d}}\left(r / R_{\mathrm{disk}}\right)^{-15 / 8} \exp \left(-\frac{\pi}{4}\left[\frac{z}{h(r)}\right]^{2}\right), \\
h(r) & =R_{\mathrm{disk}} H_{0}\left(r / R_{\mathrm{disk}}\right)^{9 / 8} \\
\rho_{\text {disk }}^{\mathrm{F} 2}(r, z) & =\rho_{\mathrm{d}}\left(r / R_{\mathrm{disk}}\right)^{-\alpha} \exp \left(-\left|\frac{z}{h(r)}\right|\right), \\
h(r) & =R_{\text {disk }} H_{0}\left(r / R_{\mathrm{disk}}\right)^{\beta}
\end{aligned}
$$

where $R_{\text {disk }}$ is the outer disk radius and $H_{0}$ the ratio of the disk height to the disk radius. The F1 form is most widely used for YSO disk models; however, this form does not guarantee a better fit to all observations, and an alternative form of F2 is also exceptionally considered (e.g. Lazareff et al. 1990; Fischer et al. 1996; Murakawa et al. 2008b). In this form, the values of $\alpha$ and $\beta$ are free parameters. The difference between these models is the concentration of the matter particularly toward the midplane. While the F1 form explicitly indicates a flared structure, the F2 form can be a flat (non-flared) structure in the case of $\beta=1$. The mass density coefficient $\rho_{\mathrm{d}}$ is determined with the specified disk mass $M_{\text {disk }}$, assuming a gas-to-dust mass ratio of 100 . We set the inner disk radius $R_{\text {in }}$ to be $5 \mathrm{AU}$, which is approximated as the dust sublimation radius for the assumed luminosity. Under hydrostatic equilibrium, the disk height is correlated with the pressure, the mass density, and the viscosity (Shakura \& Sunyaev 1973; Pringle 1981; Chiang \& Goldreich 1997). However, in our analysis, such physical quantities are either not clearly determined or not given from observations. Thus, the $H_{0}$ is parameterized and is determined by fits of SED and intensity and polarization images.

For the envelope, we use the model of the slowly rotating, infalling envelope (Ulrich 1976; Cassen \& Moosman 1981; Terebey et al. 1984). The mass density distribution is given by

$\rho_{\mathrm{env}}(R, \mu)=\frac{\dot{M}_{\mathrm{env}}}{4 \pi \sqrt{G M_{\star} R^{3}}}\left(1+\frac{\mu}{\mu_{0}}\right)^{-1 / 2}\left(\frac{\mu}{\mu_{0}}+\frac{2 \mu_{0}^{2} R_{\mathrm{c}}}{R}\right)^{-1}$,

where $R, G, \dot{M}_{\mathrm{env}}$, and $R_{\mathrm{c}}$ are the radial distance from the central star $R=\sqrt{r^{2}+z^{2}}$, the gravitational constant, the envelope mass infall rate, and the centrifugal radius, respectively. The quantity $\mu$ is the cosine of the polar angle $\mu=\cos \theta$, and $\mu_{0}$ is the angle of a stream line as related through the equation $\mu_{0}^{3}+\mu_{0}\left(R / R_{\mathrm{c}}-1\right)-\mu\left(R / R_{\mathrm{c}}\right)=0$. The polar cavity is the region for $\mu>\mu_{0}$. The density inside the cavity is reduced to 0.01 compared to the surrounding envelope. From the large-scale optical images, the full opening angle of the NGC 2261 nebula was measured as $\sim 80^{\circ}$ (Lightfoot 1989). We set the $\mu_{0}$ to be $\cos \left(80^{\circ} / 2\right)$. The centrifugal radius $R_{\mathrm{c}}$ is set to be $R_{\text {disk }}$; that is a region inside $R_{\text {disk }}$ is regarded as the disk and outside as the envelope. Although clear definitions of the disk and envelope exist in theory, these are in fact difficult to distinguish. We estimate $R_{\text {disk }}$ from our radiative transfer modeling. The extension of the nebula is $4^{\prime}$ (Lightfoot 1989), which corresponds to $\sim 0.93 \mathrm{pc}$. We apply this value for the outer radius $R_{\text {out }}$. 
The absorption and scattering properties depend on various properties of the dust, such as the chemical composition, grain size distribution, and surface micro-structure. However, our goal is to investigate the grain size. We adopted spherical grains with the DL-chemistry (Draine \& Lee 1984) to make it simpler. The dust was assumed to have an MRN-like size distribution (Mathis et al. 1977); $0.005 \mu \mathrm{m} \leq a \leq a_{\max } \mu \mathrm{m}$ and $n(a) \propto a^{-3.5}$. We applied multiple dust models; i.e. grains with different $a_{\max }$ for the disk and envelope. The $a_{\max }$ in the envelope was found to be $0.25 \mu \mathrm{m}$ from a one-dimensional single scattering model based on our previous Subaru/CIAO data (M08a). However, this model produces a slightly higher polarization than the VLT/NACO data in the $K_{\mathrm{S}}$ band. Thus, we chose $0.5 \mu \mathrm{m}$, which results in an intermediate value. The disk was divided into three mass density domains with the thresholds of mass density $\rho_{\text {th }}$, and a different $a_{\max }$ was applied for each domain. In our modeling, we determine the $a_{\max }$ in the disk and the $\rho_{\text {th }}$ values.

\subsection{SED and polarimetric data}

The photometric data in the $U B V R I J H K L M N Q$ bands are from Lorenzetti et al. (1983), Cohen (1973), Hillenbrand et al. (1992), and Pezzuto et al. (1997). The MIR to FIR photometric data are from the Midcourse Space Experiment (MSX) point source catalog and the Infrared Astronomical Satellite (IRAS) point source catalog. The 10-20 $\mu \mathrm{m}$ spectra are from the IRAS low-resolution spectra (LRS). The submillimeter and millimeter photometric data are from Mannings (1994). The typical aperture sizes of the above photometric data are $6^{\prime \prime}-12^{\prime \prime}$ in the infrared and $15^{\prime \prime}-$ $22^{\prime \prime}$ otherwise. Because the extension of the nebulosity $\left(\sim 4^{\prime}\right)$ is much greater than these aperture sizes, the measured fluxes are lower than the total fluxes. We have confirmed this aperture size effect and find that factors of up to 2-3 lower fluxes in the optical, submillimeter, and longer wavelength ranges. On the other hand, these are less than a factor of 2 in the NIR to FIR, because a large fraction of fluxes are localized towards the central region. Since our interest is the disk instead of the outer envelope, we ignored the aperture size effect in the resulting SED. However, the interstellar extinction should be taken into account because $\mathrm{R}$ Mon is located towards the galactic plane $\left(l=+01^{\circ} .27\right)$. We corrected the flux assuming the standard galactic extinction of $1.6 \mathrm{mag} \mathrm{kpc}^{-1}$ (Allen 1973).

For the polarimetric data, we use our Subaru/CIAO data in the $J$ and $H$ bands and VLT/NACO data in the $K_{\mathrm{S}}$ band (M08a). The estimated point-spread function (PSF) sizes of these data are $0 . ' 54,0.35$, and 0. '066 in the $J, H$, and $K_{\mathrm{S}}$ bands, respectively. The model images are convolved with the Gaussian functions of the equivalent FWHM sizes to compare with the observations.

\subsection{Results}

To perform radiative transfer calculations, we used our own Monte Carlo STSH code, which can compute the SED, the dust temperature, and the Stokes IQUV images of models with arbitrary geometries (up to three-dimension) and multiple dust models (Murakawa et al. 2008b). We first tried some parameter sets to find approximate solutions and appropriate parameter grids and ranges to scan. The parameters and their ranges follow. For $\alpha$ and $\beta$ of the F2 disk form, we chose $(\alpha, \beta)=(1.5,1.0)$, which fits the nebulous feature towards the central star and the appearance of the polarization disk better than $(15 / 8,9 / 8)$. If an outer disk radius of $1500 \mathrm{AU}$, which was estimated from a gas phase observation by F06, is applied, the apparent size of the polarization disk becomes slightly smaller than the observed size. We find that $3000 \mathrm{AU}$ is better. This size is, in fact, the same as an extension of the central feature detected at $2.7 \mathrm{~mm}$ (Fuente et al. 2003), although they considered that this feature may include the envelope. The inclination angle $\theta_{\text {inc }}$ of the disk was estimated to be $70^{\circ} \pm 10^{\circ}$ measured from the polar axis (Close et al. 1997). We found that this is quite reasonable. Thus, we adopted $\theta_{\text {inc }}$ as $70^{\circ}$. For grain sizes, we tried $a_{\max , 1}$ of $100.0,1000.0$, and $10000.0 \mu \mathrm{m}$ in the highest density region of $\rho \geq \rho_{\mathrm{th}, 1}$ and $a_{\mathrm{max}, 2}$ of 1.0 and $10.0 \mu \mathrm{m}$ in the intermediate density region of $\rho_{\text {th }, 2} \leq \rho \leq \rho_{\text {th }, 1}$. The examined threshold densities were $\rho_{\text {th }, 1}$ of $5 \times 10^{-21} \mathrm{~g} \mathrm{~cm}^{-3}$ and $1 \times 10^{-20} \mathrm{~g} \mathrm{~cm}^{-3}$ and $\rho_{\text {th }, 2}$ of $1 \times 10^{-21} \mathrm{~g} \mathrm{~cm}^{-3}$ and $3 \times 10^{-21} \mathrm{~g} \mathrm{~cm}^{-3}$. For others, we examined the ratio of the height to the radius of the disk $H_{0}$ of $0.1,0.2,0.3,0.4,0.5$, and 1.0 , the disk mass of $0.01,0.02,0.03$, and $0.05 M_{\odot}$, and the mass infalling rate $\dot{M}_{\text {env }}$ of $1 \times 10^{-6}$ to $1 \times 10^{-5} M_{\odot} \mathrm{yr}^{-1}$.

We examined 2880 parameter sets. Determining the bestfitmodel is an important process. One can apply an objective method such as $\chi^{2}$ test. This kind of analysis is preferred, for example, for fitting spectral data, such as the $10 \mu \mathrm{m}$ silicate feature. However, when different types of data are simultaneously taken into account, as in our model, the situation is different. In general, the best SED-fit model does not guarantee fitting the best in the intensity images or the polarization data, and vise versa. Even if we attempt to apply $\chi^{2}$ test, it is not clear how to determine the weights for SED, intensity distribution, and polarization data without a subjectivity. In addition, we did not intend to reproduce all aspects of the observations. Thus, we fit by eye and chose a good solution. We believe that our selected model can be somewhat different the best model without changing our conclusion. The parameters of the selected model have a disk mass of $0.02 M_{\odot}$, a disk scale height of 0.4 , a mass infalling rate of $5 \times 10^{-6} M_{\odot} \mathrm{yr}^{-1}$, an $a_{\max , 1}$ of $1000.0 \mu \mathrm{m}$, a $\rho_{\text {th, } 1}$ of $1 \times 10^{-20} \mathrm{~g} \mathrm{~cm}^{-3}$, an $a_{\mathrm{max}, 2}$ of $10.0 \mu \mathrm{m}$, and a $\rho_{\mathrm{th}, 2}$ of $1 \times 10^{-21} \mathrm{~g} \mathrm{~cm}^{-3}$. The optical depth of the disk is 4.2 at $V$, which is calculated by integrating radially outward through the entire disk $R_{\text {disk }}$ in the midplane $(z=0)$. Table 1 summarizes all parameters and the uncertainty of the corresponding parameters. In later discussions, we justify and present the uncertainties of important disk properties: a large radius of $3000 \mathrm{AU}$, an intermediate disk height of $H_{0}=0.4$, extremely large grains of $a_{\max }=1000.0 \mu \mathrm{m}$, and an intermediate optical depth of $\tau=4.2$ in the disk midplane. In the next section, we discuss them in detail and briefly describe how the uncertainties of some parameters listed in Table 1 are derived.

Figure 1a shows the absorptive opacity $\kappa$, the scattering opacity $\sigma$, and the total opacity of the selected dust models as functions of the wavelength. In the dust models of $a_{\max }=0.5 \mu \mathrm{m}$ and $10.0 \mu \mathrm{m}$, the opacities suddenly change in the NIR and MIR, respectively, where the size parameter $x=2 \pi a / \lambda$ becomes $\sim 1$. On the other hand, a nearly flat spectrum is seen in the $a_{\max }=$ $1000.0 \mu \mathrm{m}$ model. Figure 1c presents the central region of the mass density distribution of the selected model. The two contour lines denote the density thresholds of $\rho_{\mathrm{th}, 1}$ and $\rho_{\mathrm{th}, 2}$. Because of our assuming mass density domains, millimeter-sized grains exist even up to $\sim 100 \mathrm{AU}$ above the midplane. According to theories of grain growth and dust settling (e.g. Miyake \& Nakagawa 1993; Dullemond \& Dominik 2004b; Schräpler \& Henning 2004; Dullemond \& Dominik 2005; Tanaka et al. 2005), such extremely large grains could sediment closer to the midplane. In a future work, one can examine the distribution of large grains in disks with a large radius and an intermediate optical depth. The dust temperature is $z 60 \mathrm{~K}$ in the whole disk and has a nearly cylindrical distribution (see Fig. 1d). 
Table 1. Parameters of our radiative transfer model of R Mon.

\begin{tabular}{|c|c|c|}
\hline Parameters & Adopted values & Comments $^{1}$ \\
\hline \multicolumn{3}{|c|}{ Stellar parameter } \\
\hline$T_{\star}$ & $30000 \mathrm{~K}$ & 2 \\
\hline$L_{\star}^{\star}$ & $1400 L_{\odot}$ & 3 \\
\hline$M_{\star}$ & $10.4 M_{\odot}$ & 2 \\
\hline$d$ & $800 \mathrm{pc}$ & 4 \\
\hline$R_{\star}$ & $9.7 \times 10^{10} \mathrm{~cm}$ & calculated \\
\hline \multicolumn{3}{|c|}{ dust grains (common) } \\
\hline chemistry & DL-chemistry & 5 \\
\hline size & $\begin{array}{l}0.005 \leq a \leq a_{\max } \mu \mathrm{m} \\
n(a) \propto a^{-3.5}\end{array}$ & 6 \\
\hline \multicolumn{3}{|c|}{ dust grains (disk) } \\
\hline $\begin{array}{l}a_{\text {max, } 1} \\
\text { region }\end{array}$ & $\begin{array}{l}1000.0 \mu \mathrm{m} \\
\rho_{\text {th }} \geq 1 \times 10^{-20} \mathrm{~g} \mathrm{~cm}^{-3}\end{array}$ & $100.0-1000.0$ \\
\hline $\begin{array}{l}a_{\max , 2} \\
\text { region }\end{array}$ & $\begin{array}{l}10.0 \mu \mathrm{m} \\
\rho_{\text {th }} \geq 1 \times 10^{-21} \mathrm{~g} \mathrm{~cm}^{-3}\end{array}$ & $\gtrsim 1.0$ \\
\hline $\begin{array}{l}a_{\max } \\
\text { region }\end{array}$ & $\begin{array}{l}0.5 \mu \mathrm{m} \\
\rho_{\mathrm{th}} \leq 1 \times 10^{-21} \mathrm{~g} \mathrm{~cm}^{-3}\end{array}$ & adopted \\
\hline \multicolumn{3}{|c|}{ dust grains (envelope) } \\
\hline$a_{\max }$ & $0.5 \mu \mathrm{m}$ & adopted \\
\hline & disk & \\
\hline$R_{\text {in }}$ & $5 \mathrm{AU}$ & 7 \\
\hline$R_{\text {disk }}$ & $3000 \mathrm{AU}$ & estimated \\
\hline$M_{\text {disk }}$ & $0.02 M_{\odot}$ & $0.02-0.03$ \\
\hline$H_{0}$ & 0.4 & $0.3-0.4$ \\
\hline$\alpha, \beta$ & $1.5,1.0$ & adopted \\
\hline$\tau$ & 4.2 & 8 \\
\hline$\theta_{\text {inc }}$ & $70^{\circ}$ & \\
\hline \multicolumn{3}{|c|}{ envelope } \\
\hline$R_{\text {out }}$ & $0.93 \mathrm{pc}\left(=4^{\prime}\right)$ & 9 \\
\hline$\dot{M}_{\text {env }}$ & $5.0 \times 10^{-6} M_{\odot} \mathrm{yr}^{-1}$ & $3.0-5.0 \times 10^{-6}$ \\
\hline$\mu_{0}$ & $\cos \left(80^{\circ} / 2\right)$ & adopted \\
\hline$M_{\mathrm{env}}$ & $32 M_{\odot}$ & calculated \\
\hline$\tau$ & 10.6 & \\
\hline
\end{tabular}

Notes. ${ }^{(1)}$ Ranges give the uncertainty of the corresponding model parameters; ${ }^{(2)}$ Hillenbrand et al. (1992); ${ }^{(3)}$ Cohen et al. (1984); ${ }^{(4)}$ Jones \& Herbig (1982); ${ }^{(5)}$ Draine \& Lee (1984); ${ }^{(6)}$ Mathis et al. (1977); ${ }^{(7)}$ sublimation radius; ${ }^{(8)}$ the calculated $V$-band optical depth in the midplane; (9) Lightfoot (1989).

Because of an intermediate optical depth of 4.2 at $V$-band, the entire disk is moderately heated. Figure $1 \mathrm{~b}$ compares the model SED with the observations. The overall shape of the model SED agrees with the observations. The scattered light dominates in the wavelengths up to $\sim 2 \mu \mathrm{m}$. A smooth emission bump at $10 \mu \mathrm{m}$, but not a sharp triangle shape, in the observation is quantitatively reproduced in our model, which is also due to large grains in the disk (e.g. van Boekel et al. 2003, 2005). In submillimeter to millimeter wavelength ranges, we see higher fluxes by a factor up to 2-3 in our results, in which a large fraction of the flux comes from the outer envelope. The results fit better if a $20^{\prime \prime}$ aperture is applied, as mentioned in Sect. 2.1. The uncertainty in the mass infalling rate of the envelope listed in Table 1 is estimated from the above flux error. Figure 2 shows the $J, H$, and $K_{\mathrm{S}}$ band intensity and polarization images of our observations (upper panels) and the selected model results (lower panels). Except for the lobe shape, a nearly spherical nebulosity close to the central star is reproduced in the model images. Table 2 shows the result of aperture polarimetry.

Because an arc-like feature extending towards the northwest is seen in the polarization disk and this probably indicates the scattered light from the disk surface, we used a small aperture of 0.1 in circular diameter at the central star to reduce a dilution
Table 2. Result of aperture polarimetry.

\begin{tabular}{lrrr}
\hline \hline Model & $J(\%)$ & $H(\%)$ & $K(\%)$ \\
\hline observation & 7.1 & 1.5 & 1.1 \\
selected model & 1.7 & 1.6 & 1.6 \\
model G1 & 9.2 & 11.1 & 11.6 \\
model G2 & 14.1 & 21.1 & 34.2 \\
\hline
\end{tabular}

by other polarized components than from the polarization disk. The polarization values in the selected model are $1.7 \%, 1.6 \%$, and $1.6 \%$ in the $J H K$ bands, respectively, and are in good agreement with observations, excluding the $J$ band. We believe that this discrepancy comes from contamination by highly polarized scattered flux from the envelope. The butterfly-shaped polarization disk and a weak wavelength dependency of the extension are also reproduced in our model results. In the next section, we justify our estimates of the grain size and the disk geometry and show the complementarity of these analyses.

\section{Discussion}

\subsection{Grain size}

Evidence of large grains in R Mon's disk was already discovered from the detection of a low opacity spectral index $\beta$ (Fuente et al. 2003). Thus, in this section, we investigate the consistency with other methods such as SED and polarization analyses.

We evaluate two models with different grain sizes in the disk: (1) the grains with $a_{\max }=10.0 \mu \mathrm{m}$ for $\rho_{\mathrm{th}} \geq 1 \times 10^{-21} \mathrm{~g} \mathrm{~cm}^{-3}$, i.e. $a_{\max }=1000.0 \mu \mathrm{m}$ grains are replaced by the $a_{\max }=10.0 \mu \mathrm{m}$ grains in the selected model; and (2) only $a_{\max }=0.5 \mu \mathrm{m}$ grains for the entire disk, hereafter models G1 and G2, respectively. Figures 3 and 4 show the SED and the model images in the $K_{\mathrm{S}}$ band. The result of aperture polarimetry is also presented in Table 2. In the optical wavelength range, the flux is too low because smaller grains result in higher extinction in this wavelength range. The same reason stands in the NIR, although the difference in flux is much less. A slightly better fit in the G1 model is found. The real R Mon disk possibly has a slightly higher optical depth than the selected model. It is worth considering whether an optical depth of the disk in the NIR is kept constant for different dust models. Such a model SED probably satisfies the observations, as intended. However, we should also take the disk mass into account (some relative discussions, Chiang \& Goldreich 1997; D’Alessio et al. 2001; Wolf et al. 2003; Murakawa et al. 2010). Because the optical depth $\tau$ is approximately proportional to $(\sigma+\kappa) M_{\text {disk }}$, changing the dust size has a strong impact on the resulting disk mass. With the selected dust model of $a_{\max }=1000.0 \mu \mathrm{m}$, a disk mass of $0.02 M_{\odot}$ is required, which roughly agrees with an estimated value of $\sim 0.01 M_{\odot}$ from an observation of gas phase $\mathrm{CO}$ emission lines (F06). In contrast, the G1 and G2 models have $\sim 100$ and $\sim 10$ times higher dust opacities in the optical, causing $\sim 100$ and $\sim 10$ times lower disk masses. Thus, small grain models are inadequate.

Detection of a flux excess in the submillimeter and millimeter wavelength ranges is regarded as evidence of large grains (e.g. Beckwith \& Sargent 1991), because large grains have a low opacity spectral index $\beta$ (Draine 2006, and references therein). It is known that the $\beta$ is 2 for the interstellar population and $<1$ for extremely large grains. In our dust models, the $\beta$ values for the $a_{\max }=1000.0 \mu \mathrm{m}$ and $a_{\max }=10000.0 \mu \mathrm{m}$ are 1.3 and 1.0 , respectively, at a wavelength of $1.3 \mathrm{~mm}$, which are reasonable 

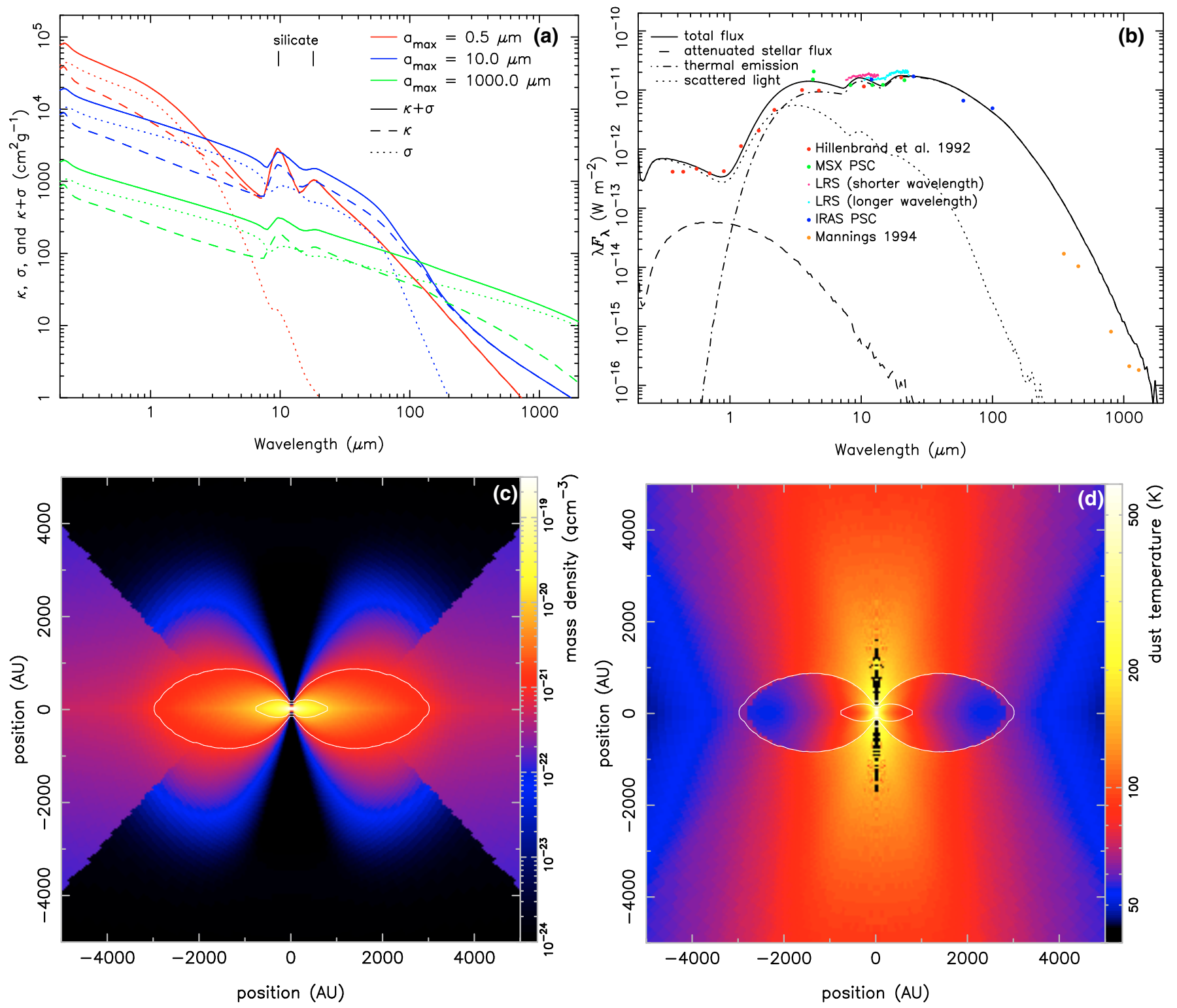

Fig. 1. Results of the selected model. a) The dust opacities as functions of the wavelength. The red, blue, and green curves denote the dust models with $a_{\max }$ values of $0.5 \mu \mathrm{m}, 10.0 \mu \mathrm{m}$, and $1000.0 \mu \mathrm{m}$, respectively. The dotted, dashed, and solid lines denote the scattering opacity $\sigma$, the absorptive opacity $\kappa$, and the total opacity $\kappa+\sigma$, respectively. b) Comparisons of the model SEDs with the previous observations. The solid, dashed, dasheddotted, and dotted curves denote the total flux, the attenuated stellar flux, the thermal emission, and the scattered flux. The red, green, orange, light blue, violet, and yellow dots are data from Hillenbrand et al. (1992), the MSX point source catalog (PSC), the IRAS LRS spectra of short and long wavelengths, IRAS PSC, and Mannings (1994). c, d) The mass density and dust temperature distribution maps in the inner $5000 \times 5000$ AU region. The two contour lines indicate the threshold densities of $1 \times 10^{-20} \mathrm{~g} \mathrm{~cm}^{-3}$ and $1 \times 10^{-21} \mathrm{~g} \mathrm{~cm}^{-3}$ to change the grain sizes. From the inner, higher density region, the grain models with $a_{\max }=1000.0 \mu \mathrm{m}, 10.0 \mu \mathrm{m}$, and $0.5 \mu \mathrm{m}$ are applied.

values compared to similar ones calculated by Draine (2006, see Fig. 3). In contrast, F06 find a low value of 0.3. It is very likely that a compact feature detected at $1.3 \mathrm{~mm}$ towards the central star $(\sim 150 \mathrm{AU})$ is the only region to contribute the millimeter flux. Therefore, the $\beta$ should be characterized by large dust grains existing in this region. However, the dust opacity with this low $\beta$ is significantly lower than the $a_{\max }=1000.0 \mu \mathrm{m}$ model. As a result, a much higher disk mass $\left(\gg 0.01 M_{\odot}\right)$ is required to fit the optical to NIR fluxes, but this is unrealistic. This low $\beta$ issue still remains puzzling in $\mathrm{R}$ Mon. The most plausible reason for this discrepancy lies in the different assumptions. For example, F06 assume a disk temperature of $215 \mathrm{~K}$ and a mean opacity of $\kappa=0.01\left(\lambda_{\mathrm{mm}} / 1.3\right)^{-\beta} \mathrm{cm}^{2} \mathrm{~g}^{-1}$, but these are not in our modeling. In addition, when the $\beta$ is estimated from the submillimeter/millimeter flux slope $\alpha$, a compensation term $\Delta$ should be taken into account, i.e. $\alpha \approx 2+\beta /(1+\Delta)$, as discussed in Sect. 4.1 in a paper by Beckwith \& Sargent (1991). It is obvious that a more self-consistent model is preferable for more qualitative study.

The grain-size effect also appears in the intensity and polarization images. The nebulosities are more asymmetric in both G1 and G2 models compared to the selected model because of the higher opacities in the smaller grains. The grain size effect is more prominent in the polarization data. In the G1 model, the appearance of the polarization disk is acceptable, but the polarizations of $\sim 10 \%$ in the $J H K$-bands are too high. In the G2 model, a strong $\mathrm{X}$-shaped feature with high polarization is seen, and the result of an aperture polarimetry shows low (14\% at $J$ ) to high 

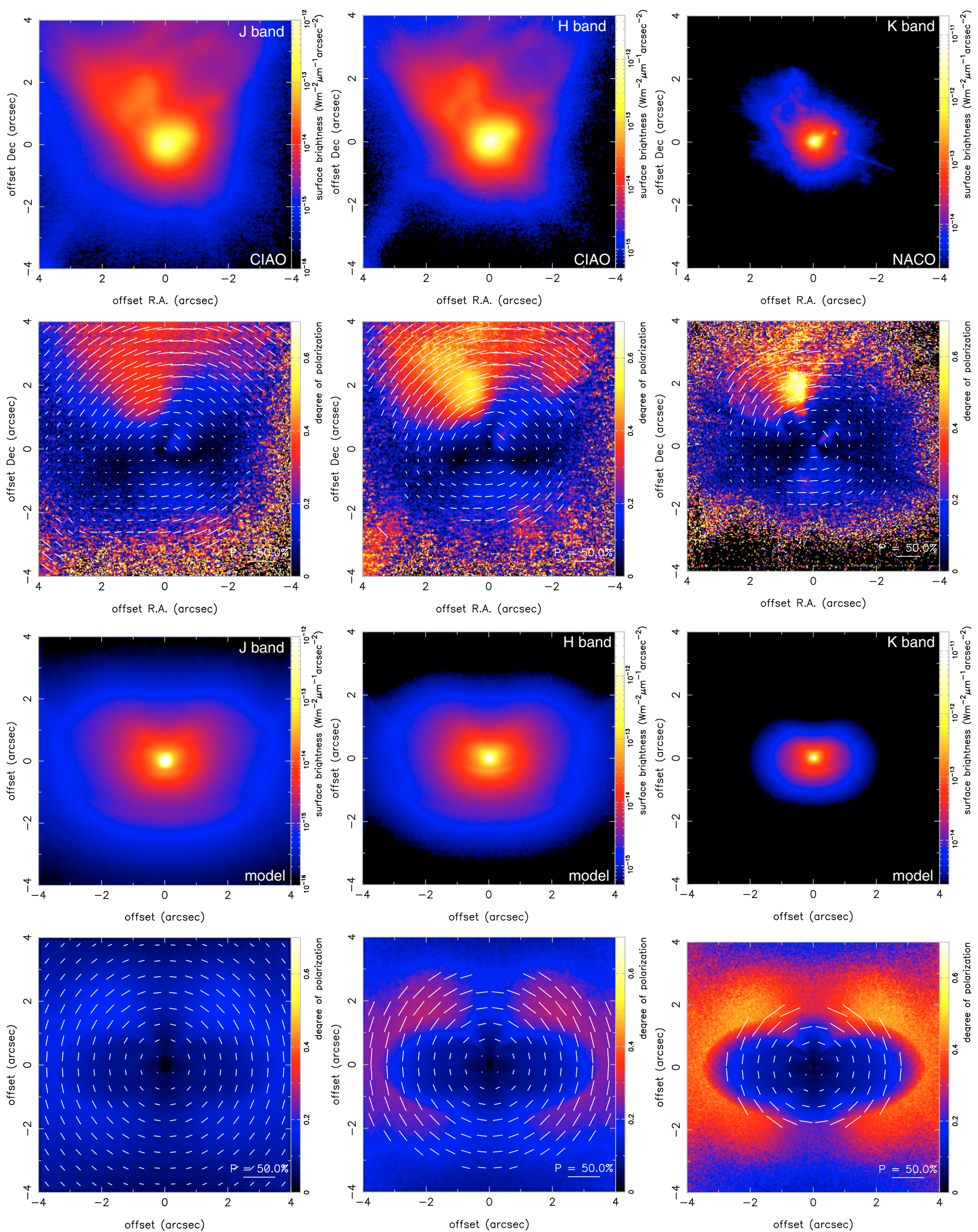

Fig. 2. The intensity and polarization images in the $J, H$, and $K_{\mathrm{S}}$ bands. The polarization vector lines are overlaid on the polarization images. The upper two rows show our previous observational results (Murakawa et al. 2008a). The $J H$-band data and the $K_{\mathrm{S}}$-band data are the results of the Subaru/CIAO and VLT/NACO observations, respectively. The bottom two rows are our selected model results. In these results, Gaussian functions that have the corresponding PSF sizes are convolved. 


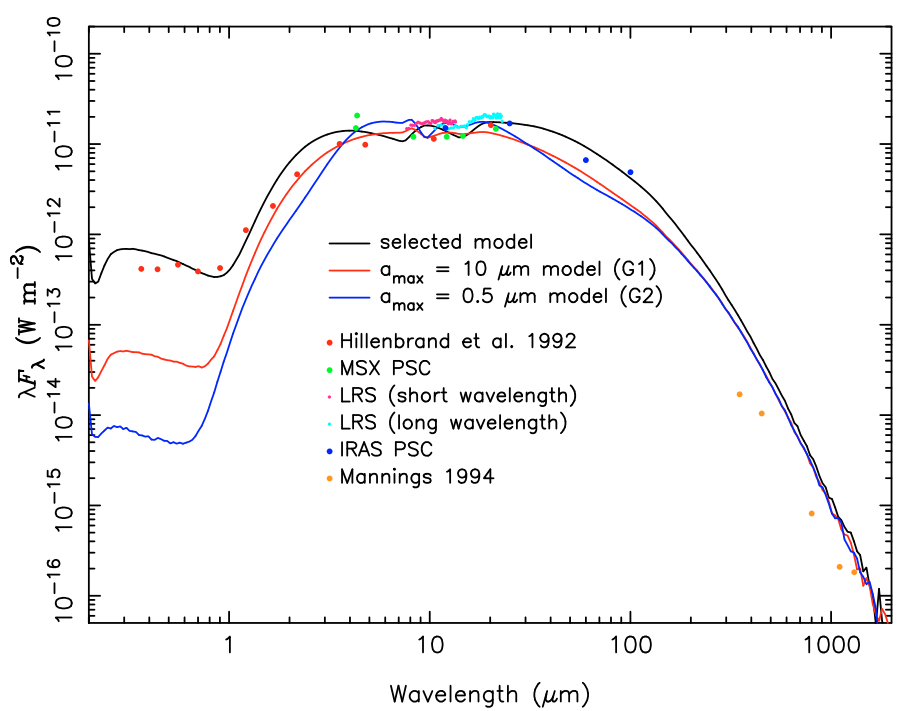

Fig. 3. Comparison of model SEDs with different grain sizes in the disk. The black, red, and blue lines denote the results of the selected model, model G1 $\left(a_{\max }=10.0 \mu \mathrm{m}\right.$ grains for $\left.\rho \geq 1 \times 10^{-21} \mathrm{~g} \mathrm{~cm}^{-3}\right)$, and model $\mathrm{G} 2\left(a_{\max }=0.5 \mu \mathrm{m}\right.$ grains for the entire disk and envelope), respectively. All other parameters are the same as the selected model.

(34\% at $K$ ) polarizations, which is the opposite wavelength dependence to typical T Tauri stars. Strictly speaking, the grain shape (i.e. asphericity and fluffiness) and the alignment efficiency should be taken into account for the aperture polarimetry. The dichroism (or deferential opacity $\Delta Q \equiv Q_{/ /}-Q_{\perp}$ ) appears when the dust size parameter $x=2 \pi a / \lambda$ is around unity, i.e. a submicron size in the NIR. However, a spherical grain approximation would be enough if large grains $(x \gg 1)$ are expected. The polarization analysis also rules out small grain models.

\subsection{Disk geometry}

We examined three models with (1) an F1 disk form of Eq. (1), (2) a small disk radius of $100 \mathrm{AU}(=0.125$ at $800 \mathrm{pc})$, and (3) a high disk height $\left(H_{0}=1.0\right)$, hereafter the D1, D2, and D3 models. For the D1 model, we set an $H_{0}$ of 0.7 , a disk mass of $0.01 M_{\odot}$, and threshold mass densities of $\rho_{\text {th }, 1}=3 \times 10^{-21} \mathrm{~g} \mathrm{~cm}^{-3}$ and $\rho_{\text {th, } 2}=3 \times 10^{-22} \mathrm{~g} \mathrm{~cm}^{-3}$ to fit the SED. For the D2 model, we set a disk scale height of 0.1 and a disk mass of $0.01 M_{\odot}$. We applied only the $a_{\max }=1000.0 \mu \mathrm{m}$ dust model in the entire disk. This disk geometry is typical of T Tauri and Herbig Ae stars and is similar to the ones estimated from the observations at $1.3 \mathrm{~mm}$ continuum (F06) and a previous radiative transfer model (Alonso-Albi et al. 2009). For the D3 model, the disk height is from previous $\mathrm{CO}$ emission line observations and modeling (F06). The other parameters are the same as the selected model. Figures 5 and 6 show the model results of the SED and the images in the $K_{\mathrm{S}}$ band.

The D1 model result is actually fairly good. However, the nebulosity around the central star is slightly more asymmetric and the MIR flux too low compared to the selected model and the observations. Such a slight asymmetric appearance of the nebulosity is caused by a higher optical depth due to a higher $\alpha$ value of $15 / 8$ compared to the selected model (1.5). A similar discussion is found in Fischer et al. (1996). We believe that a lower $\alpha$ value (e.g. 1.5) is better for the R Mon disk. For the other aspects, such as whether a flaring $(\beta>1)$ or flat $(\beta=1)$ structure or whether the inner rim is puffed up or not, we do not find any clear clue in our modeling.

In the D2 model, the intensity image has a cup-like appearance, which is often seen in T Tauri stars, but not in the case of $\mathrm{R}$ Mon. Such a feature with a dark lane is produced by a high optical depth in the disk midplane. In this case, the matter of a $0.01 M_{\odot}$ is condensed in a $100 \mathrm{AU}$ radius. This high optical depth model provides another problem for fluxes that are too low in the optical and NIR. To solve this problem, one can decrease the disk mass. However, as already discussed in the last section, this contradicts an estimation of the disk mass with gas phase data. A model by Alonso-Albi et al. (2009) applies a disk radius and a dust disk mass of $150 \mathrm{AU}$ and $1.4 \times 10^{-4} M_{\odot}$, which corresponds to a total mass of $0.014 M_{\odot}$ if a typical gas-to-dust mass ratio of 100 is assumed. Their model SED fits the observation closely. Unfortunately, the intensity and polarization images do not satisfy the observation because their model has a dust-free region between the outer disk boundary at $150 \mathrm{AU}$ and the innermost part of the outer envelope at $700 \mathrm{AU}$. In the intensity and polarization images, a feature of this gap should appear. We interpret that their modeled disk is the inner part of the disk, where large grains exist, instead of the entire disk. The polarization image provides another important clue to distinguish it from compact disks. With such a disk, the polarization disk looks like the hands of a clock at 8:20 rather than a disk, and a strong X-shaped feature is produced, which is not seen in R Mon.

The D3 model fits the SED and the intensity image well. However, the appearance of the polarization disk looks spherical instead of butterfly-shaped as seen in the observations. Thus, the dust disk is expected to be geometrically thinner than the gas disk. Also considering that R Mon's disk has a somewhat thick geometry, it is likely that the dust layer is concentrated towards the midplane more than the gas phase molecules.

In this section, we have presented comparison models with different disk geometries. We find that even though the resulted model SEDs satisfy the observations, the intensity and polarization images do not guarantee a fit to the observations. The opposite should also be true, although we did not show it. We expect that R Mon's disk has a large radius and possibly a thinner geometry than the gas phase disk; i.e. the dust settling is ongoing.

\subsection{The polarization and dust disks of $R$ Mon}

From these results, we find that R Mon's disk is unique and contrasts with typical $\mathrm{T}$ Tauri and Herbig Ae disks. The polarization disk in our high-resolution data traces the real disk closely and the polarization vector alignment is weak. The point is that the optical depth of R Mon's disk $(\tau=4.2)$ is not expected to be as high as those of typical $\mathrm{T}$ Tauri disks $\left(\tau \sim 10^{5}-10^{6}\right.$, e.g. Whitney et al. 2004). Such large differences in the optical depth come from large differences in the disk volumes but comparable disk masses. R Mon's disk has an outer radius of 3000 AU and a disk height $H_{0}$ of 0.4 , while those of typical T Tauri stars are $\sim 100$ AU and 0.01-0.1, respectively; that is R Mon's disk is 1000 to 10000 times bigger. In contrast, the disk masses are comparable $\left(\sim 0.01 M_{\odot}\right)$. Because of this difference in optical depth of the disk, an alternative interpretation of the polarization disk other than the BM mechanism, should be considered.

In the BM mechanism, the direct star light and singlescattered light vanish, but the double-scattered light can reach the observer and causes a vector alignment. Because photons behave as if they avoid the optically thick midplane, we call this phenomenon the roundabout effect (M10). In addition, the second scattering event can happen outside the disk, and the 

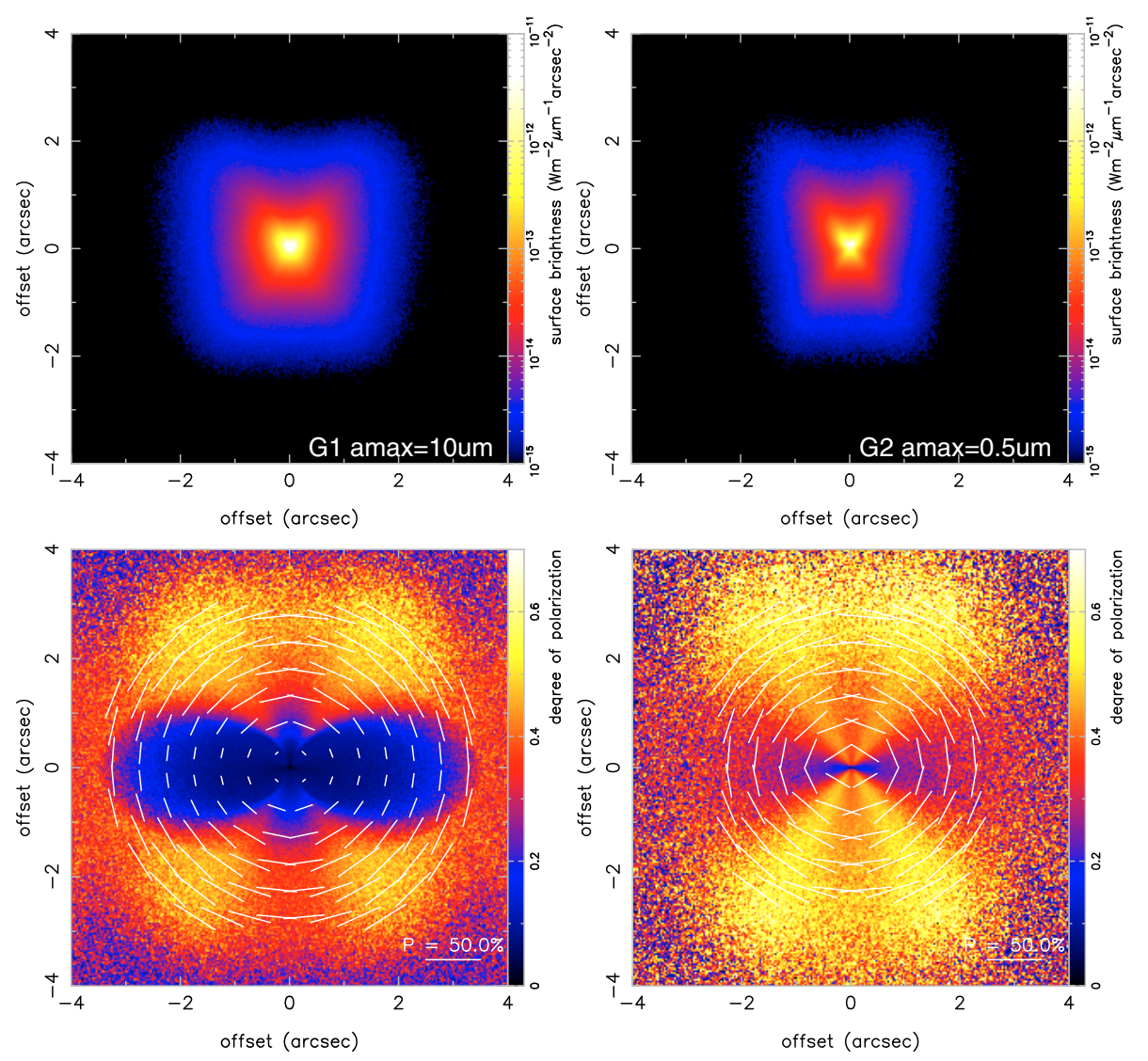

Fig. 4. The model results with different grain sizes in the disk. The left and right columns correspond to the models $\mathrm{G} 1$ and $\mathrm{G} 2$, respectively. The wavelength is $2.20 \mu \mathrm{m}$.

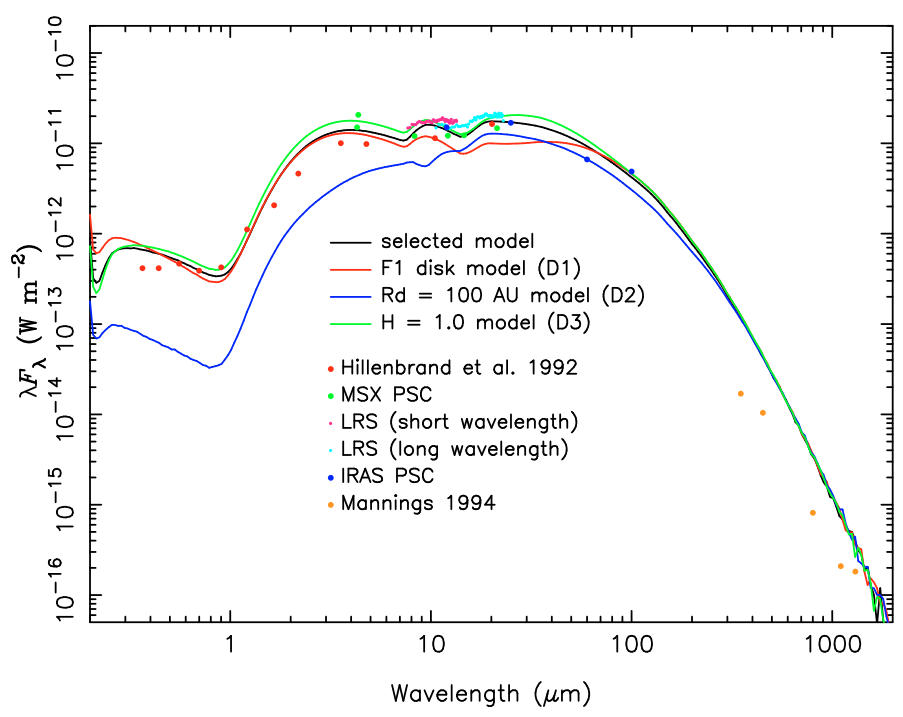

Fig. 5. Comparison of model SEDs with different disk geometry parameters. The black, red, blue, and green lines denote the results of the selected model, the $\mathrm{F} 1$ disk model (D1), the $R_{\text {disk }}=100 \mathrm{AU}$ model (D2), and the $H_{0}=1.0$ model (D3), respectively.

apparent size of the polarization disk can be larger than the real disk (M10). The scenario is different in R Mon's disk and is explained with our new model (M10). The roundabout effect is weak, and the single-scattered light still reaches the observer. In this condition, the polarization status somewhat shows the single-scattering properties of dust in the disk, such as the grain size and the disk height, which determines the scattering angle. It is worth mentioning that, although the scattering properties do not change for large grains $(x \gg 1)$ (see Fig. 1 in our previous paper M10), the result of aperture polarimetry is different between our selected model and the G1 model. In both cases, the photon penetrate the disk. However, the roundabout effect is stronger in the G1 model because the optical depth of the disk is higher for a higher dust opacity model. The flux towards the central star contains more highly polarized, scattered light from the disk surface, where small grains exist than does our selected model. As a result, a higher polarization is obtained in the G1 model. Furthermore, the projection of the real disk appears as the polarization disk, and the vector pattern looks more centrosymmetric than linearly aligned.

Here, we would like to recall the definition of a polarization disk. BM argue that the polarization disk is a region where the vectors are aligned along the equatorial plane. The polarization in this region is lower than in the outer lobe or envelope in general. At the same time, another low polarization region with nearly centro-symmetric vector alignment is often detected around the polarization disk; for example, a feature of the hands of a clock is seen in the D2 model. These features are sometimes difficult to distinguish in the observations. However, we have pointed out that the polarization disk contains important information on the grain size and/or grain shape. In contrast, the other is simply reproduced by depolarization due to multiple scattering and only indicates the existence of a high-density region near the central star (M10). Thus, we call the latter region a polarization shadow (or polarization dark lane) and distinguish it from the polarization disk.

We stress that the R Mon is a special case. According to this definition, the butterfly-shaped low-polarization feature with $\sim 4^{\prime \prime}$ extension towards the central star appears to be a polarization shadow because the vectors are not horizontally aligned. Nevertheless, we still regard this feature as the polarization disk 

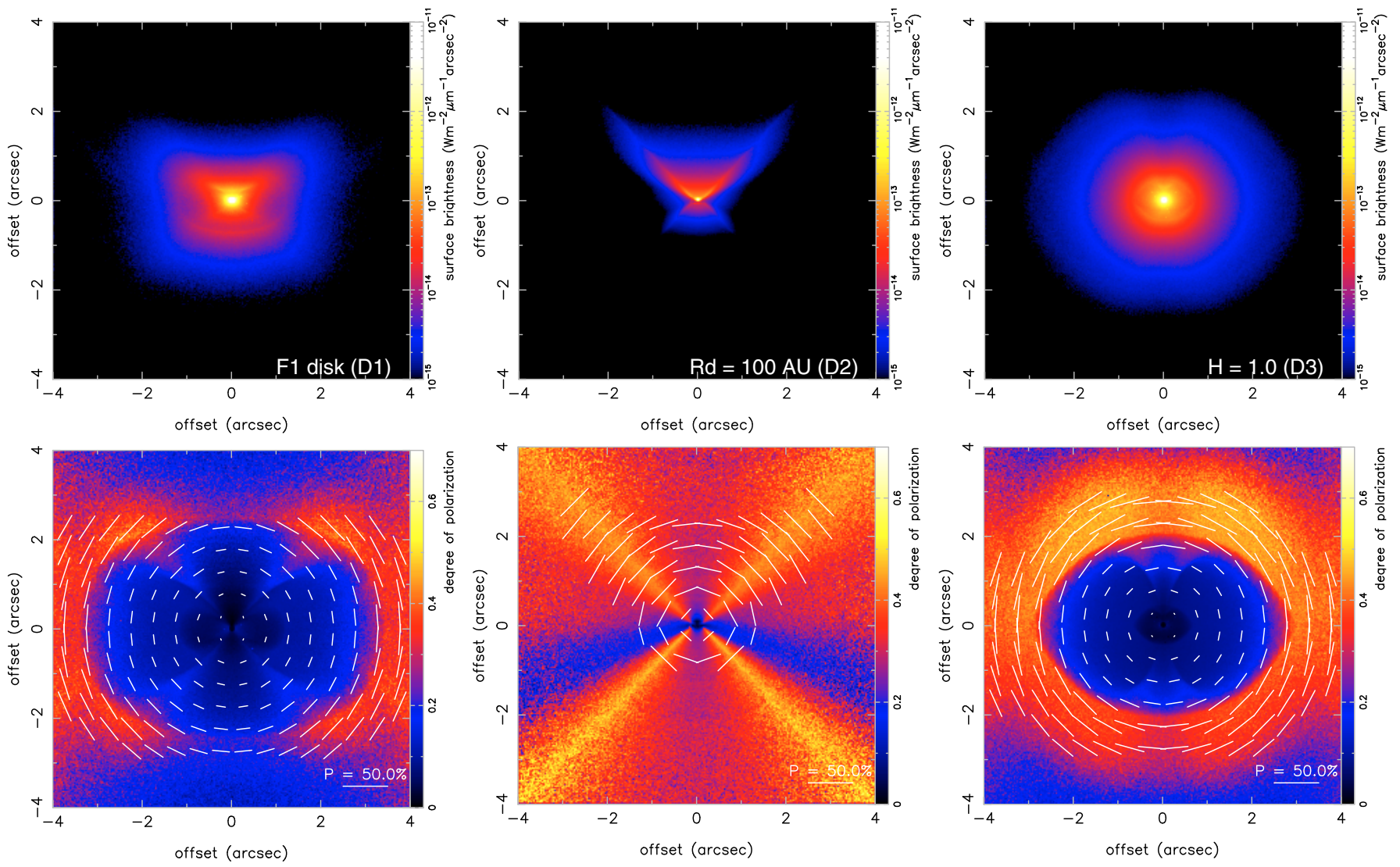

Fig. 6. Model results with different disk geometries. From the left, the results in the $K_{\mathrm{S}}$ band of the F1 disk form (D1), a small disk radius of $100 \mathrm{AU}(=0$ ' 125$)$ (D2), and an $H_{0}$ of 1.0 (D3) are presented. The disk scale height of the D3 model is the same as the one estimated from the gas phase disk (F06).

because it turns out from our modeling that this feature traces well the projected angular size of the real dust disk. A low NIR polarization and not detecting the vector alignment indicate the presence of large grains and the intermediate NIR optical depth of the disk.

The disk size is also different from typical $\mathrm{T}$ Tauri disks and more similar to ones in some massive protostars such as IRAS 20126+4104 (1000 AU, Sridharan et al. 2005), AFGL 490 (in a transition stage to Herbig Be, 1500 AU, Schreyer et al. 2006), M 17 (3000 AU, Steinacker et al. 2006), and CRL 2136 (2000 AU, Murakawa et al. 2008b). Theoretical calculations predict that a thousand $\mathrm{AU}$ size can form round a $10 M_{\odot}$ star (e.g. Yorke \& Bodenheimer 1999). On the other hand, the expected large dust grains in R Mon's disk agrees with T Tauri disks. For massive protostars, estimation of the grain sizes is less precise or is done with comparably fewer samples, because it is difficult from only an SED fit and from a detection of millimeter flux excesses. However, in some cases, high NIR polarizations are detected (e.g. Whittet et al. 2008; Murakawa et al. 2008b). In particular, for an object where the central feature is visible, such as CRL 2136, a high NIR polarization can be reproduced with only submicron-sized grains in the disks despite the grain shape. Therefore, in terms of the disk geometry and grain sizes, $\mathrm{R}$ Mon's disk shows an intermediate property between $\mathrm{T}$ Tauri stars and massive protostars. Furthermore, it is also interesting that ongoing dust settling is expected in the R Mon disk, as mentioned in the previous section. Some authors have investigated the disk evolution, both dust settling and dust growth (e.g. Miyake \& Nakagawa 1993; Dullemond \& Dominik 2004b; Schräpler \& Henning 2004; Dullemond \& Dominik 2005;
Tanaka et al. 2005). The grain growth time $t_{\text {grow }}$, in which a $0.1 \mu \mathrm{m}$ dust grain grows to micron size, is expected to be shorter than the formation time of the dust layer $t_{\text {settle, }}$, (e.g. $t_{\text {grow }} \sim$

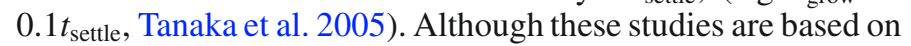
$\mathrm{T}$ Tauri disks, a similar conclusion is still applicable in R Mon's disk.

\section{Conclusion}

We have modeled the $\mathrm{R}$ Mon dust disk by means of radiative transfer calculations. In our approach, we combined the polarization disk analysis with an SED fit. In addition, we used previous results from $\mathrm{CO}$ emission line observations in the radio frequencies and modeling to constrain the dust size and to compare them with the dust disk geometry. The estimated disk parameters are a disk of $3000 \mathrm{AU}$, a disk height of $H_{0}=0.4$, an optical depth in the midplane of 4.2 at $V$, a disk mass of $0.02 M_{\odot}$, and a grain size of $a_{\max }=1000.0 \mu \mathrm{m}$. We justified these parameters with some alternative models and showed how different analytical methods constrain them. For the grain size, an SED fit, a detection of the millimeter flux excess, and a polarization analysis suggest that large grains should exist in the disk. Taking practical factors into account such as observation precisions, the millimeter flux excess, and polarization analyses, can provide a quantitative evaluation, and the SED fit combined with the dust and gas phase models allows a qualitative estimation in any size range. For the disk geometry, even if it is possible to satisfy the SED with a small radius of $\sim 100-150$ AU, the NIR intensity and polarimetric images do not permit this and require a large radius. We believe that such a small radius, which is detected at a radio 
continuum of $2.7 \mathrm{~mm}$ (F06), is the inner part of the disk where large grains exist.

We attempted to apply our generalized scattering model to interpreting R Mon's polarization disk. Because of a large outer disk radius and an intermediate disk height, it turned out that the polarization disk roughly traces the projected real dust disk. Not detecting a vector alignment and a nearly constant, low NIR polarizations $(P \sim 1-2 \%)$ are explained similarly, where the roundabout effect is weak. From these results, we found that R Mon's disk is unique and contrasts typical T Tauri and Herbig Ae disks. The large disk radius is fairly similar to those of some massive (proto)stars. In R Mon's disk, the dust grains grow in size, and the dust condensation towards the midplane is probably currently in progress because an intermediate disk height is expected. This agrees with a theoretical prediction that the dust settling time is longer than the dust growth time.

We have demonstrated that our complementary combination method (polarization analysis, SED fit including the dust and gas phase modeling) is a powerful technique for deriving important properties of YSO disks. A multiple analytical methods based on different physics will be important in the coming ELT, HERSCHEL, JWST, and ALMA era.

Acknowledgements. Our NIR polarimetric images were obtained at the European Southern Observatory (proposal ID: 078.C-0554) and the Subaru telescope. We would like to thank an anonymous referee for his/her meaningful suggestions and comments.

\section{References}

Adams, F. C., \& Shu, F. H. 1986, ApJ, 308, 836

Alonso-Albi, T., Fuente, A., Bachiller, R., et al. 2009, A\&A, 497, 117

Allen, C. W. 1973, Astrophysical Quantities, 3rd ed. (London: Athlone Press)

Bastien, P., \& Ménard, F. 1988, ApJ, 326, 334

Bastien, P., \& Ménard, F. 1990, ApJ, 364, 232

Beckwith, S. V. W., \& Sargent, A. I. 1991, ApJ, 381, 250

Bertout, C., Basri, G., \& Bouvier, J. 1988, ApJ, 330, 350

Calvet, N., Hartmann, L., Kenyon, S. J., \& Whitney, B. A. 1994, ApJ, 434, 330

Cassen, P., \& Moosman, A. 1981, Icarus, 48, 353

Chiang, E. I., \& Goldreich, P. 1997, ApJ, 490, 368

Close, L. M., Roddier, F., Hora, J. L., et al. 1997, ApJ, 489, 210

Cohen, M. 1973, MNRAS, 161, 97

Cohen, M., Harvey, P. M., Wilking, B. A., \& Schwartz, R. D. 1984, ApJ, 278, 671

D’Alessio, P., Calvet, N., \& Hartmann, L. 2001, ApJ, 553, 321

Draine, B. T. 2006, ApJ, 636, 1114

Draine, B. T., \& Lee, H. M. 1984, ApJ, 285, 89
Dullemond, C. P., \& Dominik, C. 2004a, A\&A, 417, 159 Dullemond, C. P., \& Dominik, C. 2004b, A\&A, 421, 1075 Dullemond, C. P., \& Dominik, C. 2005, A\&A, 434, 971

Dullemond, C. P., Dominik, C., \& Natta, A. 2001, ApJ, 560, 957

Fischer, O., Henning, Th., \& Yorke, H. W. 1994, A\&A, 284, 187

Fischer, O., Henning, Th., \& Yorke, H. W. 1996, A\&A, 308, 863

Fuente, A., Rodríguez-Franco, A., Testi, L., et al. 2003, ApJ, 598, L39

Fuente, A., Alonso-Albi, T., Bachiller, R., et al. 2006, ApJ, 649, L119

Hillenbrand, L. A., Strom, S. E., Vrba, F. J., \& Keene, J. 1992, ApJ, 397, 613

Isella, A., \& Natta, A. 2005, A\&A, 438, 899

Jones, B. F., \& Herbig, G. H. 1982, AJ, 87, 1223

Kenyon, S. J., \& Hartmann, L. 1987, ApJ, 323, 714

Kenyon, S. J., Whitney, B. A., Gomez, M., \& Hartmann, L. 1993, ApJ, 414, 773

Lazareff, B., Pudritz, R. E., \& Monin, J.-L. 1990, ApJ, 358, 170

Lightfoot, J. F. 1989, MNRAS, 239, 665

Lorenzetti, D., Saraceno, P., \& Strafella, F. 1983, ApJ, 264, 554

Lucas, P. W., \& Roche, P. F. 1997, MNRAS, 286, 895

Lucas, P. W., \& Roche, P. F. 1998, MNRAS, 299, 699

Mannings, V. 1994, MNRAS, 271, 587

Mathis, J. S., Rumple, W., \& Nordsieck, K. H. 1977, ApJ, 217, 425

Meeus, G., Waters, L. B. F. M., Bouwman, J., et al. 2001, A\&A, 365, 476

Men'shchikov, A. B., Henning, T., \& Fischer, O. 1999, ApJ, 519, 257

Miyake, K., \& Nakagawa, Y. 1993, Icarus, 106, 20

Murakawa, K. 2010, A\&A, in press (M10)

Murakawa, K., Preibisch, T., Kraus, S., et al. 2008a, A\&A, 488, L75 (M08a)

Murakawa, K., Preibisch, T., Kraus, S., \& Weigelt, G. 2008b, A\&A, 490, 673

Murakawa, K., Ueta, T., \& Meixner, M. 2010, A\&A, 510, 30

Natta, A., Prusti, T., Neri, R., et al. 2001, ApJ, 371, 186

Pezzuto, S., Strafella, F., \& Lorenzetti, D. 1997, ApJ, 485, 290

Pringle, J. E. 1981, ARA\&A, 19, 137

Schräpler, R., \& Henning, T. 2005, ApJ, 614, 960

Schreyer, K., Semenov, D., Henning, Th., \& Forbrich, J. 2006, ApJ, 637, L129

Shakura, N. I., \& Sunyaev, R. A. 1973, A\&A, 24, 337

Sridharan, T. K., Williams, S. J., \& Fuller, G. A. 2005, ApJ, 631, L73

Steinacker J., Chini R., Nielbock M., et al. 2006, A\&A, 456, 1013

Tanaka, H., Himeno, Y., \& Ida, S. 2005, ApJ, 625, 414

Terebey, S., Shu, F. H., \& Cassen, P. 1984, ApJ, 286, 529

Testi, L., Natta, A., Shepherd, D. S., \& Wilner, D. J. 2003, A\&A, 403, 323

Ulrich, R. K. 1976, ApJ, 210, 377

van Boekel, R., Waters, L. B. F. M., Dominik, C., et al. 2003, A\&A, 400, L21

van Boekel, R., Min, M., Waters, L. B. F. M., et al. 2005, A\&A, 437, 189

Walsh, J. R., \& Malin, D. F. 1985, MNRAS, 217, 31

Whitney, B. A., \& Hartman, L. 1993, ApJ, 402, 605

Whitney, B. A., Kenyon, S. L., \& Gomez, M. 1997, ApJ, 485, 703

Whitney, B. A., Indebetouw, R., Bjorkman, J. E., \& Wood, K. 2004, ApJ, 617, 1177

Whittet, D. C. B., Shenoy, Hough, J. H., Lazarian, A., \& Hoang, T. 2008, ApJ, 674,304

Wilner, D. J., D’Alessio, P., Calvet, N., Claussen, M. J., \& Hartmann, L. 2005, ApJ, 626, L109

Wolf, S., Padgett, D., \& Stapelfeldt, K. R. 2003, ApJ, 588, 373

Wood, K., Kenyon, S. J., Whitney, B., \& Turnbull, M. 1998, ApJ, 497, 404

Yorke, H. W., \& Bodenheimer, P. 1999, ApJ, 525, 330 